\title{
Aplicações da Psicoeducação no Contexto da Saúde
}

\author{
Carina Belomé Lemes ${ }^{1}$ \\ Escola de Enfermagem da Universidade Federal do Rio Grande do Sul, \\ Porto Alegre, RS, Brasil \\ Secretaria Estadual de Saúde do Rio Grande do Sul, Porto Alegre, RS, Brasil \\ Jorge Ondere Neto \\ Universidade Federal do Rio Grande do Sul, Porto Alegre, RS, Brasil \\ Pontifícia Universidade Católica do Rio Grande do Sul, Porto Alegre, RS, Brasil
}

\section{Resumo}

A psicoeducação é uma técnica que relaciona os instrumentos psicológicos e pedagógicos com objetivo de ensinar o paciente e os cuidadores sobre a patologia física e/ou psíquica, bem como sobre seu tratamento. Assim, é possível desenvolver um trabalho de prevenção e de conscientização em saúde. O presente trabalho tem como objetivo investigar o uso da psicoeducação no âmbito da saúde relatando a sua aplicabilidade nas doenças físicas e mentais, bem como seu envolvimento nessa área. O método consiste em uma revisão sistemática da literatura cuja palavra-chave utilizada foi "psicoeducação" nas bases de dados LILACS (Literatura Latino-Americana e do Caribe em Ciências da Saúde), PePSIC (Periódicos Eletrônicos de Psicologia) e SciELO (Scientific Electronic Library Online). Em um total de 59 artigos, foram incluídos 29. Destes, 9 artigos eram repetidos, resultando em um total de 20 estudos. A psicoeducação pode ser utilizada tanto para transtornos psicológicos quanto para doenças orgânicas, correspondendo a $85 \%$ e $15 \%$ dos artigos, respectivamente. Ela pode ser aplicada tanto em cuidadores quanto em pacientes, correspondendo a $40 \%$ e $45 \%$ dos estudos, respectivamente. A sua técnica pode ser aplicada nos seguintes contextos: cuidados paliativos, doenças crônicas, grupoterapia e saúde pública. A partir dos resultados e da discussão, a psicoeducação é uma técnica que possui aplicações em diversas áreas da saúde, englobando transtornos psicológicos e doenças orgânicas.

Palavras-chave: Psicoeducação, intervenção, saúde.

\section{Applying Psychoeducation in Health}

\begin{abstract}
Psychoeducation is an approach that relates psychological and pedagogical tools having as its objective the teaching directioned to patients and caregiver about physical and/or psychic pathology and its treatment as well. Thus, it is possible to develop a prevention and awareness work in health. The present work has as its aim to investigate the usage of psychoeducationin the area of health relating its application in mental and physical illnesses and its involvement in the area as well. The method consists of a systematic review of literature whose keyword is "psycho education", and its results were found in the following data basis: LILACS (Literatura Latino-Americana e do Caribe em Ciências da Saúde), PePSIC (Periódicos Eletrônicos de Psicologia) and SciELO (Scientific Electronic Library Online). Totalizing 59 articles, only 29 were used. It is possible to conclude that psycho education can be used for
\end{abstract}

Endereço para correspondência: Avenida Anita Garibaldi, 2381, Boa Vista, Porto Alegre, RS, Brasil. Fones: (051) 999595949 / (051) 999595914.E-mail: ninablemes@gmail.com e j.ondere@gmail.com 
both psychological disorders and organic illnesses, as in $85 \%$ and $15 \%$ of the articles respectively. It can be applied for both caregivers (30\% of the studies) and patients ( $45 \%$ of the studies). Its technique can be practiced in the following contexts: palliative care, chronic diseases, group therapy, and public health. From the results and the discussion it is possible to notice that psycho education is a technique that contains applications in many health areas, covering psychological disorders and organic illnesses.

Keywords: Psychoeducation, intervention, health.

\section{Aplicaciones de la Psicoeducación en el Contexto de la Salud}

\section{Resumen}

Psicoeducación es una técnica que relaciona las herramientas psicológicas y pedagógicas con el fin de enseñar a los pacientes y cuidadores sobre la condición física y/o psíquica, así como su tratamiento. Así que se puede desarrollar un trabajo de prevención y de concienciación sobre la salud. Este estudio pretende investigar el uso de la psicoeducación en salud informando sobre su aplicabilidad en las enfermedades físicas y mentales, así como su participación en este ámbito. El método consiste en una revisión sistemática de la literatura, cuya palabra clave "psicoeducación" fue buscada en las siguientes bases de datos: LILACS (Literatura Latino-Americana e do Caribe em Ciências da Saúde), PePSIC (Periódicos Eletrônicos de Psicologia) y SciELO (Scientific Electronic Library Online). En un total de 59 artículos fueron utilizados 29. De estos, nueve artículos se repitieron, dando lugar a un corpus de 20 estudios. Por lo tanto se concluyó que la psicoeducación puede ser utilizada tanto para los trastornos psicológicos cuanto para enfermedades orgánicas, que corresponde a $85 \%$ y el $15 \%$ de los artículos, respectivamente. Se puede aplicar tanto a los cuidadores (30\% de los estudios) y a los pacientes ( $45 \%$ de los estudios). Su técnica se puede practicar en estos contextos: cuidados paliativos, enfermedades crónicas, terapia de grupo y salud pública. A partir de los resultados y de la discusión, parece que la psicoeducación es una técnica que tiene aplicaciones en muchas áreas de la salud, que abarca trastornos psicológicos y enfermedades orgánicas.

Palabras clave: Psicoeducación, intervención, salud.

A psicoeducação é uma intervenção psicoterapêutica a qual tem como objetivo enfocar mais as satisfações e ambições relacionadas aos objetivos almejados pelo paciente do que uma técnica voltada para curar determinada doença (Authier, 1977). Segundo o autor, a psicoeducação propiciou uma maneira de auxiliar o tratamento das doenças mentais a partir das mudanças comportamentais, sociais e emocionais cujo trabalho permite a prevenção na saúde. Dessa forma, a psicoterapia iniciou um processo de ter um caráter também educativo tanto para o paciente quanto para seus cuidadores cujo objetivo é ensiná-los sobre o seu tratamento psicoterápico para que possam ter consciência e preparo para lidar com as mudanças a partir de estratégias de enfrentamento, fortalecimento da comunicação e da adaptação (Bhattacharjee et al., 2011). Assim, a maneira mais efetiva para auxiliar as pessoas é ensiná-las a se ajudarem, propiciando conscientização e autonomia (Authier, 1977).

O modelo psicoeducacional envolve diferentes teorias psicológicas e educativas, além disso, utiliza dados teóricos de outras disciplinas como a educação, a filosofia, a medicina e entre outras com intuito de ampliar o fornecimento de informações ao paciente para que obtenha um entendimento não fragmentado acerca de seu diagnóstico (Cole \& Lacefield, 1982). Sendo assim, as informações teóricas de distintas disciplinas são fornecidas no sentido de que o paciente possa obter um entendimento holístico de seu padecimento. Também não há apenas um ambiente em que a psicoeducação possa ser empregada, ela pode ser utilizada em instituições hospitalares, ambulatoriais, militares, industriais 
e educacionais (Cole \& Lacefield, 1982). Inclusive, o uso da psicoeducação, como recurso terapêutico, pode ser realizado via internet (Wood, Brendro, Fecser, \& Nichols, 1999). Os autores também destacam que, além das diferentes áreas disciplinares e instituições em que a psicoeducação pode ser utilizada, é possível aplicá-la em diferentes problemáticas como: terapia de luto, comportamento passivo-agressivo, ansiedade infantil, redução de estresse e etc.

A Psicoeducação teve seu início em 1970, surgindo como um modelo que envolve o paradigma da complexidade da espécie humana, nesse caso, ela envolve distintas disciplinas e teorias que podem ser inter-relacionadas para compreender e aplicar suas técnicas frente ao adoecimento do indivíduo (Wood et al., 1999). No entanto, a ressalva dos autores é que esse modelo, o qual pretende englobar as diferentes dimensões do humano, não pode ser aplicado de qualquer maneira, ou seja, deve haver um método sistemático com aplicação de testes e de técnicas específicas para averiguar qual é o procedimento psicoeducativo que possibilita resultados positivos. Isso justifica o desenvolvimento de projetos e de pesquisas acerca da psicoeducação desde seu surgimento até hoje, onde buscam explorar as evidências de sua eficácia como técnica psicoterapêutica.

Wood et al. (1999) sugerem separar o termo, a parte psico se refere ao âmbito das teorias e técnicas psicológicas existentes; a educação, por sua vez, está relacionada à área pedagógica a qual envolve o processo de ensino-aprendizagem. Nesse caso, a psicoeducação engloba o desenvolvimento social, emocional e comportamental do sujeito, sendo que o profissional atua como um agente de mudanças, fornecendo assistências às habilidades adquiridas e propiciando práticas que tenham embasamento científico ao paciente. Sendo assim, existe a psicoeducação psicodinâmica voltada mais para os aspectos afetivos e conflitivos do sujeito, a psicoeducação comportamental a qual enfoca as mudanças comportamentais utilizando a observação do comportamento e desenvolvendo um programa de reforço ou positivo ou negativo, a psicoeducação sociológica a qual tem como proposta envolver um grupo propiciando a ele a conscientização de seus comportamentos, ideologias e valores sociais, a psicoeducação cognitivo-afetiva que engloba a relação recíproca entre o aspecto afetivo e cognitivo, a psicoeducação ecológica e a psicoeducação do desenvolvimento (Wood et al., 1999).

Em um trabalho de familiares com pacientes diagnosticados com Alzheimer, Rahmani, Ranjbar, Ebrahimi, e Hosseinzadeh (2015) realizaram uma pesquisa para investigar se a psicoeducação é uma técnica que possui eficácia. Para isso, aplicaram o Questionário OMI, que avalia as atitudes frente à pessoa com deficiência mental, em um grupo experimental antes e depois da psicoeducação; em um grupo controle, aplicaram o OMI sem realizar a intervenção. A partir dos resultados obtidos, foi possível verificar que, a partir da aplicação psicoeducativa, o grupo experimental teve um aumento de $80 \%$ em suas atitudes positivas frente ao paciente com Alzheimer, enquanto o grupo controle teve $28,57 \%$. Portanto, a psicoeducação possui dados que comprovam a sua eficácia a partir do questionário utilizado.

Por fim, há uma pesquisa que teve como objetivo investigar se a psicoeducação promove insight em pacientes com esquizofrenia (Ruzanna, Marhani, Parveen, \& Cheah, 2010). Os autores obtiveram a confirmação de diagnóstico de esquizofrenia a partir da Mini International Neuropsychiatric Interview (MINI) em 70 pacientes, então, avaliaram-nos utilizando a Schedule for the Assessment of Insight (SAI), que consiste em uma escala que investiga a capacidade de insight, antes do programa de psicoeducação. A partir da intervenção psicoeducacional, os autores aplicaram a SAI e obtiveram, como resultado, quase o dobro da capacidade de insight $(3,40$ para 6,34). Portanto, foi possível verificar, a partir dos dados da SAI, que a psicoeducação é uma técnica que possui eficácia para pacientes com diagnóstico de esquizofrenia.

Portanto, a psicoeducação é um modelo complexo, visto que envolve diferentes teorias e técnicas tanto psicológicas quanto pedagógicas voltadas ao âmbito social, comportamental e cognitivo do indivíduo, possibilitando uma 
compreensão multiperspectival a qual envolve o adoecimento. Como visto, a psicoeducação pode ser empregada em diferentes locais e problemáticas, sendo seu uso de significativa importância, pois tem como objetivo realizar prevenção, promoção e educação em saúde. Além disso, a partir dos estudos de Chien (2008) e de Rahmani et al. (2015), é possível verificar que os dados obtidos a partir dos instrumentos utilizados comprovam a eficácia da psicoeducação.

\section{Objetivo}

Investigar o uso da psicoeducação no âmbito da saúde relatando a sua aplicabilidade nas doenças físicas e mentais, bem como seu envolvimento na área da saúde.

\section{Método}

Para realizar a revisão sistemática, primeiramente, foram consultadas as bases de dados LILACS (Literatura Latino-Americana e do Caribe em Ciências da Saúde), PePSIC (Periódicos Eletrônicos de Psicologia) e SciELO (Scientific Electronic Library Online) utilizando como palavras-chave o conceito "psicoeducação" levando em consideração "todos os campos", dessa forma, não se fez seleção apenas pelo título e etc. O período estabelecido foi de 2010 a 2014, englobando os últimos cinco anos. Como critério de inclusão, estabeleceu-se (a) artigos tanto teóricos quanto empíricos de língua portuguesa, (b) estar dentro do período mencionado acima; (c) ter como investigação a psicoeducação como técnica psicoterapêutica; (d) apresentar resultados; (e) ter ou doença ou quadro psicopatológico envolvidos; (e) descrever para quem é aplicada a psicoeducação, no caso, o público-alvo, ou seja, ou familiar, ou cuidador, ou o próprio paciente. Por fim, após esse processo descrito, os artigos foram inclusos a partir da leitura do título e do resumo para, então, ler na íntegra aqueles que corresponderam com os critérios conforme o estabelecido. Nesse caso, artigos sobre psicoeducação que não tiveram como enfoque ou doença ou quadro psicopatológico e que não demonstraram resultados de sua aplicação foram exclu- ídos, visto que não cumprem com o objetivo do presente trabalho.

$\mathrm{Na}$ base de dados da LILACS foram encontrados um total de 26 artigos, destes, foram incluídos 12 na amostra, pois os demais não estão dentro do período de cinco anos. Na base PePSIC, obteve-se 13 artigos sendo que 8 foram excluídos pelo mesmo motivo citado. Já na base SciELO, foram encontrados 20 artigos e 9 foram excluídos também em função da data. Assim, em um total de 59 artigos, foram incluídos 29. Destes, 9 eram repetidos, resultando em um total de 20 estudos para serem discutidos no presente trabalho.Vale destacar que os quadros de Alzheimer e demência foram englobados em uma mesma categoria denominada "Disfunções Cognitivas", visto que, a partir da leitura, estão relacionados com quadro cuja cognição é o enfoque do distúrbio.

\section{Resultados}

Em relação aos autores dos artigos encontrados na revisão sistemática, os dois trabalhos sobre transtorno afetivo bipolar tem Menezes e Mello e Souza como pesquisadores da temática; já nas duas revisões de literatura - uma relacionada à demência e a outra ao estresse dos cuidadores de pacientes com transtorno psiquiátrico - há Sampaio como mesmo pesquisador. Também, em relação às disfunções cognitivas, os autores Lopes e Cachioni (2012, 2013a, 2013b) possuem três trabalhos relacionados aos cuidadores de pessoas com esse diagnóstico. Por fim, Wielenska é um autor presente nos dois artigos de estudo de caso. Em relação aos demais trabalhos, os autores são diferentes entre si, demonstrando uma diversidade de teóricos envolvidos com o tema.

Quanto ao delineamento metodológico, nove artigos são quantitativos (45\%), dois qualitativos $(10 \%)$, cinco revisões de literatura (25\%) e um relato de experiência (5\%). Os artigos demonstram que o uso do método quantitativo é mais prevalente nos estudos cuja psicoeducação é investigada.

A partir da revisão sistemática realizada, os artigos utilizam a técnica de psicoeducação para: 
Tabela 1

Fluxograma da Revisão Sistemática

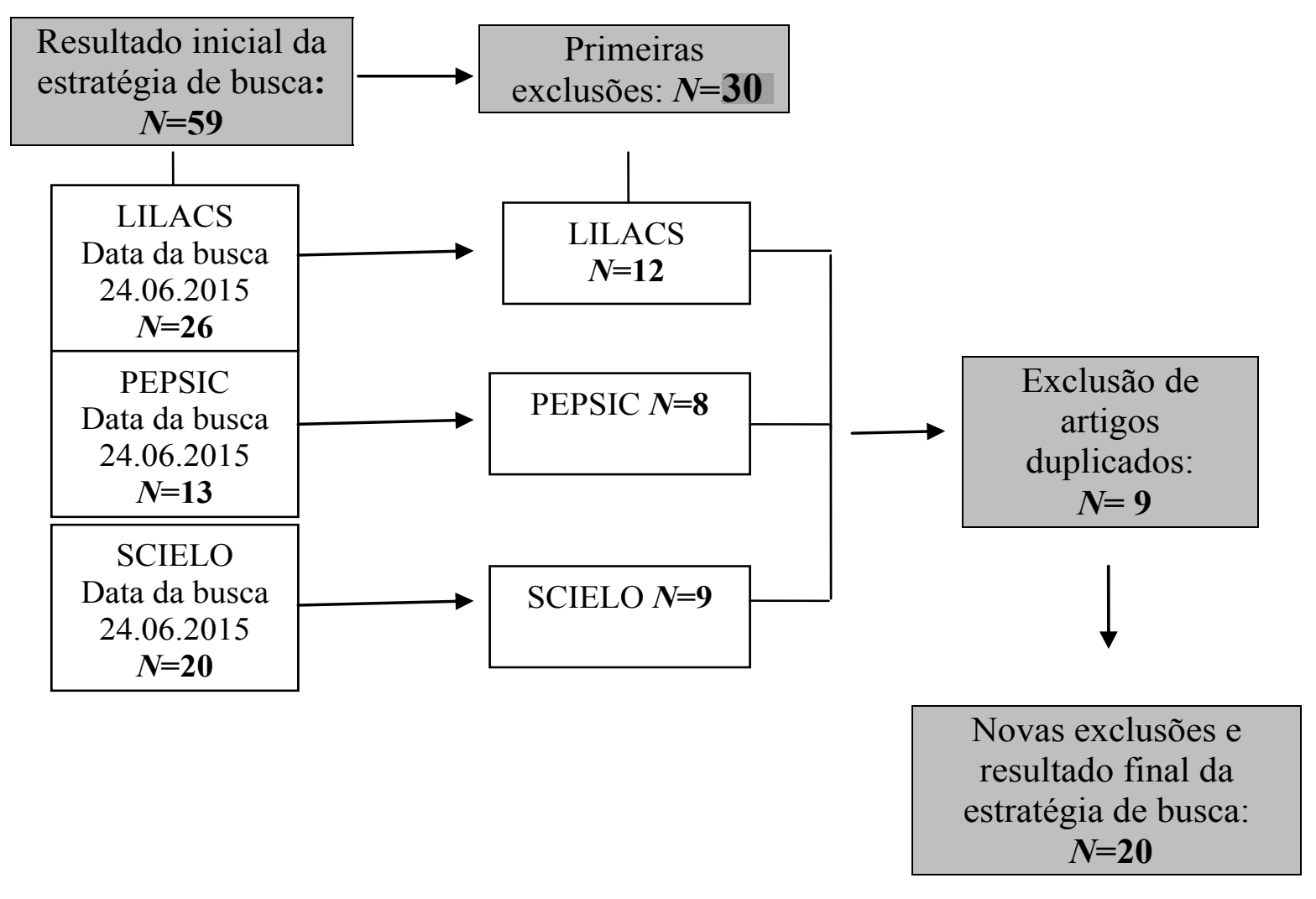

Câncer (CA; Alcântara et al., 2013); Dependência Química (Andretta \& Oliveira, 2011); Disfunção Cognitiva (Gonçalves-Pereira \& Sampaio, 2011; Lopes \& Cachioni, 2012, 2013a, 2013b; Sampaio, 2011); Doença Cardiovascular (Gomes \& Pergher, 2010); Esquizofrenia (Sousa et al., 2012); Estresse (Tabeleão et al., 2014); Fobia de Deglutição (Scemes et al., 2011); Hipertensão (HÁ; Lima-Silva \& Yassuda, 2012); Síndrome da Fadiga Crônica (Wielenska \& Banaco, 2010); Transtorno Afetivo Bipolar (TAB; Menezes \& Mello e Souza, 2011, 2012; Mussi et al., 2013); Transtorno de Ansiedade (Oliveira, 2011; Petersen, 2011); Transtorno de Estresse Pós-Traumático (TEPT; Gonçalves et al., 2010); e Transtornos Psiquiátricos (Ponciano et al., 2009). A partir disso, pode-se perceber a diversidade de quadros tanto psicopatológicos e patológicos em que a psicoeducação pode ser empregada como recurso psicoterapêutico, demonstrando sua amplitude frente às demandas do sofrimento tanto físico quanto mental.
Referente ao uso da psicoeducação para paciente e cuidador, 9 artigos (45\%) enfocam o paciente, 8 artigos (40\%) o cuidador e $3(15 \%)$ o paciente e o cuidador. Portanto, a psicoeducação pode ser utilizada tanto para pacientes quanto para cuidadores.

Pode-se, também, destacar a utilização da psicoeducação como sendo de uso exclusivo no sentido de apenas ela ter sido utilizada como intervenção psicoterapêutica (Alcântara et al., 2013; Andretta \& Oliveira, 2011; Lopes \& Cachioni, 2013a, 2013b; Menezes \& Mello e Souza, 2011, 2012; Mussi et al., 2013); diferentemente dos trabalhos os quais ela é inclusa como sendo uma das técnicas do conjunto que compõem o tratamento como um todo (Gomes \& Pergher, 2010; Gonçalves et al., 2010; Lima-Silva \& Yassuda, 2012; Oliveira, 2011; Petersen, 2011; Scemes et al., 2011; Wielenska \& Banaco, 2010). Independentemente do uso ou exclusivo ou em conjunto com outras técnicas, os resultados destes estudos identificaram um retorno positivo da 
intervenção psicoeducacional, demonstrando sua boa eficácia mesmo tendo somente ela como técnica psicoterapêutica.

Possivelmente, seu uso exclusivo se deve pelo motivo de estar sendo empregada em um estudo científico que tem como objetivo analisar apenas o uso da psicoeducação, diferentemente de uma psicoterapia a qual é composta por di- ferentes técnicas empregadas ao longo das sessões. Na psicoterapia para hipertensos, ela é utilizada junto com o treino cognitivo, sendo esta intervenção mista pouco pesquisada no campo acadêmico (Lima-Silva \& Yassuda, 2012). O que se pode concluir é que a psicoeducação pode ser incluída em diferentes conjuntos de técnicas como forma de auxiliar na saúde do paciente.

\section{Tabela 2}

Resultado da Revisão Sistemática

\begin{tabular}{|c|c|c|c|c|}
\hline Autores & (Psico)patologia & Participantes & Delineamento & Resultado/Principais Resultados \\
\hline $\begin{array}{l}\text { Alcântara, } \\
\text { Chioga, Lima, } \\
\text { Lage, \& Maia } \\
\text { (2013) }\end{array}$ & Câncer & $\begin{array}{l}\text { Paciente } \\
\text { e Cuidador }\end{array}$ & Quantitativo & $\begin{array}{l}\text { - Aplicabilidade no hospital (sala } \\
\text { de espera). } \\
\text { - Desenvolvimento de estratégias } \\
\text { de enfrentamento } \\
\text { para exames invasivos. }\end{array}$ \\
\hline $\begin{array}{c}\text { Andretta \& } \\
\text { Oliveira (2011) }\end{array}$ & $\begin{array}{l}\text { Dependência } \\
\text { Química }\end{array}$ & Paciente & Quantitativo & $\begin{array}{l}\text { - Retorno positivo à saúde pública. } \\
\text { - Diminuição do uso de álcool e maconha. }\end{array}$ \\
\hline $\begin{array}{c}\text { Gomes \& } \\
\text { Pergher (2010) }\end{array}$ & $\begin{array}{c}\text { Doença } \\
\text { cardiovascular }\end{array}$ & $\begin{array}{l}\text { Paciente } \\
\text { e Cuidador }\end{array}$ & Revisão & $\begin{array}{l}\text { - Psicoeducação aliada à descoberta } \\
\text { guiada, às técnicas de relaxamento } \\
\text { e ao treino de habilidades sociais são } \\
\text { os modelos mais utilizados. } \\
\text { - Uso na Psicoprofilaxia Cirúrgica. }\end{array}$ \\
\hline $\begin{array}{l}\text { Gonçalves et al. } \\
\qquad(2010)\end{array}$ & $\begin{array}{l}\text { Transtorno } \\
\text { de Estresse } \\
\text { Pós-traumático }\end{array}$ & Paciente & Quantitativo & - Diminuição dos sintomas. \\
\hline $\begin{array}{l}\text { Lima-Silva \& } \\
\text { Yassuda (2012) }\end{array}$ & Hipertensão & Paciente & Quantitativo & $\begin{array}{c}\text {-Ganhos cognitivos. } \\
\text { - Psicoeducação como recurso } \\
\text { psicoterapêutico adicionado ao treino } \\
\text { cognitivo propiciou resultado positivo. }\end{array}$ \\
\hline $\begin{array}{c}\text { Lopes \& } \\
\text { Cachioni }(2012)\end{array}$ & Demência & Cuidador & Revisão & $\begin{array}{c}\text { - Melhora no bem-estar do cuidador. } \\
\text { - Identificação de falta de padronização } \\
\text { da abordagem quanto à estrutura, duração } \\
\text { e conteúdos. }\end{array}$ \\
\hline $\begin{array}{l}\text { Lopes \& } \\
\text { Cachioni } \\
(2013 a)\end{array}$ & Alzheimer & Cuidador & Quantitativo & $\begin{array}{l}\text { - Melhor avaliação do cuidado. } \\
\text { - Melhora tanto no aspecto psicológico } \\
\text { quanto social; no aspecto físico, } \\
\text { não houve melhora. } \\
\text { - Grupoterapia como recurso importante. }\end{array}$ \\
\hline $\begin{array}{l}\text { Lopes \& } \\
\text { Cachioni } \\
(2013 b)\end{array}$ & Alzheimer & Cuidador & Quantitativo & $\begin{array}{l}\text { - Melhora na satisfação geral com a vida. } \\
\text { - Melhora no envolvimento social. } \\
\text { - Mais presença de afetos positivos. }\end{array}$ \\
\hline $\begin{array}{l}\text { Tabeleão, } \\
\text { Tomasi, } \\
\text { \& Quevedo } \\
\text { (2014) }\end{array}$ & Estresse & Cuidador & Quantitativo & $\begin{array}{c}\text { - Programa de Psicoeducação para } \\
\text { a saúde pública. }\end{array}$ \\
\hline
\end{tabular}




\begin{tabular}{|c|c|c|c|c|}
\hline Autores & (Psico)patologia & Participantes & Delineamento & Resultado/Principais Resultados \\
\hline $\begin{array}{l}\text { Menezes \& Mello } \\
\text { e Souza (2011) }\end{array}$ & $\begin{array}{l}\text { Transtorno } \\
\text { Afetivo Bipolar }\end{array}$ & $\begin{array}{l}\text { Paciente } \\
\text { e Cuidador }\end{array}$ & $\begin{array}{l}\text { Relato de } \\
\text { Experiência }\end{array}$ & $\begin{array}{c}\text { - Autonomia e conscientização } \\
\text { da psicopatologia. }\end{array}$ \\
\hline $\begin{array}{l}\text { Menezes } \\
\& \text { Mello } \\
\text { e Souza } \\
(2012)\end{array}$ & $\begin{array}{l}\text { Transtorno } \\
\text { Afetivo Bipolar }\end{array}$ & Paciente & Qualitativo & $\begin{array}{c}\text { - Aquisição de conhecimento. } \\
\text { - Adesão ao tratamento. } \\
\text { - Mudanças positivas na vida. } \\
\text { - Novos aprendizados na experiência } \\
\text { grupal. }\end{array}$ \\
\hline $\begin{array}{l}\text { Mussi, Soares, } \\
\text { \& Grossi } \\
\text { (2013) }\end{array}$ & $\begin{array}{l}\text { Transtorno } \\
\text { Afetivo Bipolar }\end{array}$ & Paciente & Quantitativo & $\begin{array}{l}\text { - Redução significativa da mania. } \\
\text { - Melhora no domínio "Físico" na escala } \\
\text { de Qualidade de Vida. }\end{array}$ \\
\hline Oliveira (2011) & Ansiedade & Paciente & Qualitativo & $\begin{array}{l}\text { - Diminuição dos sintomas. } \\
\text { - Aplicabilidade no serviço público. }\end{array}$ \\
\hline $\begin{array}{l}\text { Gonçalves-Pe- } \\
\text { reira \& Sampaio } \\
\qquad(2011)\end{array}$ & Demência & Cuidador & Revisão & $\begin{array}{l}\text { - Uso para promoção da saúde. } \\
\text { - Aplicação no serviço público. } \\
\text { - Psicoeducação familiar. }\end{array}$ \\
\hline Petersen (2011) & $\begin{array}{l}\text { Transtorno } \\
\text { de Ansiedade }\end{array}$ & Paciente & Revisão & $\begin{array}{l}\text { - O uso da psicoeducação em conjunto } \\
\text { com outras técnicas cognitivo-comporta- } \\
\text { mentais propiciam melhora nos sintomas } \\
\text { de ansiedade de pacientes crianças. }\end{array}$ \\
\hline Sampaio (2011) & Demência & Cuidador & Revisão & $\begin{array}{l}\text { - Parece não ser efetiva. } \\
\text { - Ausência de consenso, necessitando } \\
\text { de mais investigações. }\end{array}$ \\
\hline $\begin{array}{l}\text { Scemes, Wie- } \\
\text { lenska, Savoia, \& } \\
\text { Bernik (2011) }\end{array}$ & $\begin{array}{c}\text { Fobia de } \\
\text { Deglutição }\end{array}$ & Paciente & $\begin{array}{l}\text { Estudo } \\
\text { de Caso }\end{array}$ & $\begin{array}{l}\text { - Psicoeducação englobou as contigências } \\
\text { aversivas como um todo, não } \\
\text { se restringindo apenas à fobia alimentar. } \\
\text { - Psicoeducação auxiliou na adesão } \\
\text { à técnica de exposição. }\end{array}$ \\
\hline $\begin{array}{l}\text { Sousa, Marques, } \\
\text { Curral, } \\
\text { \& Queirós (2012) }\end{array}$ & Esquizofrenia & Cuidador & $\begin{array}{l}\text { Estudo } \\
\text { Exploratório }\end{array}$ & $\begin{array}{c}\text { - Necessidade da Psicoeducação Familiar } \\
\text { para prevenir preconceitos dos familiares } \\
\text { de esquizofrênicos. }\end{array}$ \\
\hline $\begin{array}{l}\text { Ponciano, } \\
\text { Calvacanti, } \\
\text { \& Ferés-Carneiro } \\
\quad(2009)\end{array}$ & $\begin{array}{l}\text { Transtornos } \\
\text { Psiquiátricos }\end{array}$ & Cuidador & Qualitativo & $\begin{array}{l}\text { - Compartilhamento de experiências entre } \\
\text { os cuidadores permitiu apoio mútuo. }\end{array}$ \\
\hline $\begin{array}{l}\text { Wielenska } \\
\& \text { Banaco } \\
(2010)\end{array}$ & $\begin{array}{l}\text { Síndrome } \\
\text { da Fadiga } \\
\text { Crônica }\end{array}$ & Paciente & $\begin{array}{l}\text { Estudo } \\
\text { de Caso }\end{array}$ & $\begin{array}{l}\text { - Psicoeducação como recurso } \\
\text { que auxilia na psicoterapia. }\end{array}$ \\
\hline
\end{tabular}

\section{Discussão}

Conforme os resultados obtidos, a técnica psicoeducacional pode ser utilizada tanto para doenças físicas como Câncer (Alcântara et al., 2013), Doenças Cardiovasculares (Gomes \& Pergher, 2010) e Hipertensão (Lima-Silva \&
Yassuda, 2012) quanto para doenças de ordem ou psicológica ou psiquiátrica como Dependência Química (Andretta \& Oliveira, 2011), Disfunções Cognitivas (Gonçalves-Pereira \& Sampaio, 2011; Lopes \& Cachioni, 2012, 2013a, 2013b; Sampaio, 2011), Esquizofrenia (Sousa et al., 2012), Fobia de Deglutição (Scemes et al., 
2011), TAB (Menezes \& Mello e Souza, 2011, 2012; Mussi et al., 2013), TEPT (Gonçalves et al., 2010), o Transtorno de Ansiedade (Oliveira, 2011; Petersen, 2011), Transtornos Psiquiátricos (Ponciano et al., 2009) - estas de acordo com a American Psychiatric Association [APA], 2002) - e o estresse (Tabeleão et al., 2014). É perceptível um número maior de doenças psíquicas, correspondendo a $85 \%$ dos estudos, em comparação às orgânicas $(15 \%)$.

Em relação aos resultados obtidos, a revisão de literatura acerca da sobrecarga de cuidadores de idosos com demência teve neutralidade, referindo mais investigações e pesquisas na área (Sampaio, 2011). Nos estudos quantitativos de Tabeleão et al. (2014), que também investiga a sobrecarga de cuidadores, mas no contexto de transtornos psíquicos como esquizofrenia, e de Sousa et al. (2012), que investiga as atitudes estigmatizantes de familiares de pessoas com esquizofrenia, a psicoeducação não é aplicada, os autores concluem que o seu uso é necessário, mas não a utilizam. A partir destes três estudos, não é possível concluir resultados positivos nem negativos da psicoeducação; enquanto nos demais, o retorno de sua aplicação é positivo ou ao paciente ou ao cuidador.

\section{Psicoeducação nos Cuidados Paliativos}

Apesar do crescimento de tratamentos bem sucedidos, o câncer infantil é uma doença que gera um quadro de modificações no sistema e na estrutura familiar o qual envolve tanto o paciente quanto seus pais frente ao tratamento, sendo este acompanhamento motivo de desgastes aos pais (Lau et al., 2015). Por isso, o trabalho de psicoeducação realizado na sala de espera propicia um espaço para esclarecer dúvidas e aliviar angústias vivenciadas nesse processo, permitindo uma melhora no bem-estar psicológico tanto aos pais quanto ao paciente (Alcântara et al., 2013). Além disso, há uma probabilidade de o paciente não resistir ao tratamento, isso significa que ele e seus pais estão em uma situação de perda ambígua, podendo gerar um processo de enlutamento e, nesse sentido, a psicoeducação pode ser um recurso psicoterapêutico importante para que os pais possam significar essa vivência (Alcântara et al., 2013).

Os autores referem que "nessa pesquisa, $3,56 \%$ das intervenções foram picoeducativas, $o$ que possibilitou a compreensão dos pacientes e acompanhantes acerca dos procedimentos" (Alcântara et al., 2013, p. 115). No entanto, não avaliam, a partir de dados empíricos, o quanto, de fato, a psicoeducação possibilitou a compreensão dos pacientes, além disso, pode-se verificar que o uso da psicoeducação é estatisticamente baixo em comparação às outras intervenções empregadas, a saber: intervenções lúdicas $(41,18 \%)$, escuta psicológica $(16,23 \%)$, intervenções mistas $(33,86 \%)$ e intervenção psicoeducativa $(3,56 \%)$. Portanto, ressalta-se a realização de mais estudos acadêmicos nessa área para que possam, a partir de dados obtidos, comprovar a eficácia da intervenção psicoeducativa.

\section{Intervenção Psicoeducativa em Doenças Crônicas}

O uso da psicoeducação também pode ser utilizado para doenças crônicas como as Disfunções Cognitivas (Gonçalves-Pereira \& Sampaio, 2011; Lopes \& Cachioni, 2012, 2013a, 2013b; Sampaio, 2011), a Hipertensão (Lima-Silva \& Yassuda, 2012), a Síndrome da Fadiga Crônica (Wielenska \& Banaco, 2010) e a TAB (Menezes \& Mello e Souza, 2011, 2012; Mussi et al., 2013). A intervenção psicoeducacional pode ser aplicada nos cuidadores envolvidos no cuidado ao paciente crônico, dessa forma, ela propicia melhora na avaliação do cuidado, gerando aos cuidadores motivação porque percebem ganhos positivos em seu acompanhamento desempenhado (Lopes \& Cachioni, 2012, 2013a, 2013b). Para analisar a eficácia da psicoeducação em cuidadores de pacientes com Alzheimer, Lopes e Cachioni (2013a, 2013b) realizaram uma avaliação pré e pós-intervenção utilizando o Inventário de Ônus e Benefícios Associados ao Cuidado (2013a) e as Escalas de Satisfação com a Vida, de Ânimo Positivo e Negativo e de Depressão Geriátrica (2013b). Além disso, é possível pensar no uso da psicoeducação no contexto das doenças terminais 
cujos cuidados paliativos estão envoltos, fornecendo meios para que os familiares possam realizar estratégias de enfrentamento frente à finitude de seu ente, como no caso do Câncer e das Doenças Degenerativas.

Ainda no âmbito do cuidado ao cuidador, o estudo de Gonçalves-Pereira e Sampaio (2011) e de Sousa et al. (2012) utilizam o conceito de psicoeducação familiar cuja técnica é voltada especificamente para familiares que cuidam de um ente o qual se encontra em um quadro de doença ou degenerativa ou terminal. Também destacam a importância da inclusão da técnica psicoeducativa na psicologia sistêmica como recurso aos familiares que estão envoltos no adoecimento do paciente.

\section{Psicoeducação em Grupoterapia}

A psicoeducação pode ser aplicada em grupoterapia para cuidadores de pacientes com Disfunção Cognitiva (Lopes \& Cachioni, 2013a) e para pacientes com diagnóstico de TAB (Menezes \& Mello e Souza, 2012). Os autores destacam a importância da experiência grupal para o compartilhamento de aprendizado entre os pacientes, no caso do TAB. Na pesquisa qualitativa de Menezes e Mello e Souza (2012), os pacientes com diagnóstico de TAB relataram se sentir seguros para expor seus sentimentos e pensamentos sem haver julgamentos e referiram que puderam conhecer seu transtorno, questão que possibilitou maior autoconhecimento permitindo conscientização e adesão ao tratamento.

No estudo de cuidadores de pacientes com Alzheimer, realizado em forma de grupoterapia, Lopes e Cachioni (2013a), mencionados anteriormente, referem melhora nos aspectos psicológicos e sociais; no entanto, a partir da avaliação dos instrumentos empregados antes e depois da intervenção psicoeducacional, não foi constatada melhoras no âmbito físico. Nesse sentido, pode-se questionar se a psicoeducação propicia melhoras somente no adoecimento psíquico e na vulnerabilidade social, enquanto no âmbito físico não.

\section{Psicoeducação no Contexto da Saúde Pública}

Por fim, os estudos conferem a importância da utilização da psicoeducação para o âmbito da saúde pública como ferramenta para realizar a promoção de saúde aos pacientes e aos seus cuidadores (Alcântara et al., 2013; Andretta \& Oliveira, 2011; Gomes \& Pergher, 2010; Oliveira, 2011; Gonçalves-Pereira \& Sampaio, 2011; Tabeleão et al., 2014). Vale destacar o trabalho desenvolvido por Alcântara et al. (2013) que consta de um "Projeto Sala de Espera" no Centro Pediátrico do Câncer (CPC) cujo trabalho pode fornecer um retorno positivo à promoção de saúde, além disso, ocorre no contexto hospitalar.

Há, também, o trabalho de Tabeleão et al. (2014) onde foi realizado em um Centro de Atenção Psicossocial (CAPS) voltado aos cuidadores de pessoas com transtornos psiquiátricos. Neste estudo, os autores destacam a necessidade da psicoeducação voltada aos cuidadores para reduzir a sobrecarga emocional; nesse caso, ressalta-se a importância de pesquisas acadêmicas relacionadas a esse âmbito.

A intervenção psicoeducacional também pode ser utilizada como forma de realizar uma psicoprofilaxia cirúrgica no acompanhamento pré e pós-cirúrgico em pacientes submetidos aos procedimentos cardiovasculares, sendo assim, ela é utilizada no contexto da psicologia hospitalar (Gomes \& Pergher, 2010). A psicoeducação tem como objetivo explicar e esclarecer dúvidas em relação ao procedimento cirúrgico amenizando a ansiedade do paciente no período pré-operatório, pois, conforme os autores, os pacientes podem ter catastrofização em função de pensamentos distorcidos acerca da cirurgia (Gomes \& Perguer, 2010). Em relação ao período pós-operatório, os autores referem que a psicoeducação pode ser aplicada junto com treinos cognitivos para auxiliar no período de recuperação, nesse caso, ela pode ser aliada a outras técnicas cognitivas.

Por fim, ainda no contexto da saúde pública em relação à drogadição em adolescentes infratores, Andretta e Oliveira (2011) realizaram um estudo clínico aplicando a entrevista motivacio- 
nal (EM) em grupo experimental e a psicoeducação em grupo controle. Ambos grupos foram avaliados pré e pós-intervenção com o Inventário de Depressão de Beck (BDI) e de Ansiedade (BAI), o URICA que consiste em uma escala para motivação de mudança comportamental, uma ficha de dados sócio-demográficos e uma entrevista semi-estruturada. A EM foi empregada em cinco sessões utilizando o Cannabis Youth Treatment que consiste em um programa dos Estados Unidos voltado a adolescentes infratores e a psicoeducação teve duas sessões sobre informações dos efeitos da drogadição e três sessões individuais com aplicação de técnicas cognitivo-comportamentais. Após a aplicação das intervenções, realizou-se uma pós-avaliação para investigar a eficácia obtida.

A partir da discussão, as autoras afirmam que "os resultados mostraram que tanto o grupo que recebeu a EM quanto o grupo que recebeu Psicoeducação, diminuíram o consumo de drogas demonstrando que as duas técnicas podem ser aplicadas à esta população" (Andretta \& Oliveira, 2011, p. 224), ou seja, a EM e a psicoeducação demonstram evidências de eficácia para adolescentes com dependência química. Vale ressaltar que, nesta pesquisa, a EM teve maior eficácia na diminuição do uso de maconha e de tabaco; a psicoeducação, por sua vez, no álcool e na maconha. Quanto às fases de motivação para mudança de comportamento, a pré-contemplação teve diminuição em ambos os grupos. Portanto, é possível verificar que a psicoeducação possui eficácia na dependência de maconha e álcool; no entanto, sugerem-se mais pesquisas englobando outras substâncias químicas para averiguar sua eficácia. Além disso, reduz, a partida da pesquisa, a média na fase de pré-contemplação, ou seja, a psicoeducação permite maior conscientização acerca dos malefícios da dependência química, tornando o usuário mais responsável.

\section{Conclusão}

A partir da revisão sistemática da literatura, a psicoeducação é uma técnica psicológica a qual pode ser aplicada em diferentes tipos de doenças, englobando tanto às psíquicas quan- to físicas. O que se pode também concluir é a escassez de trabalhos que a utilizam em outras doenças crônicas como a artrite cujo quadro gera dor constante, em doenças de difícil adesão ao tratamento como a tuberculose e, também, em transtornos de personalidade, por exemplo.

É importante destacar que o uso da psicoeducação no âmbito da saúde engloba não somente a Psicologia, mas, também, envolve outros enfoques disciplinares, visto que a saúde também permeia os aspectos comportamentais, emocionais e sociais. Nesse sentido, a psicoeducação se faz por um modelo cuja interdisciplinaridade é uma ferramenta necessária para a intervenção, inclusive, cumprindo com o princípio da integralidade do Sistema Único de Saúde (SUS).

A intervenção psicoeducacional é importante para dar suporte e apoio ao cuidado ao cuidador. O que se pode destacar é que os estudos enfocam os cuidadores como sendo, principalmente, familiares do paciente e não profissionais da saúde, visto que estes também poderiam se beneficiar com a psicoeducação.

Por fim, o desenvolvimento de projetos e programas de psicoeducação envolvida com um tipo de doença é importante para o trabalho de promoção de saúde, propiciando um retorno positivo à saúde pública. A psicoeducação pode utilizar recursos como vídeos, áudios, panfletos, campanhas e etc. Sendo que nesses planejamentos psicoeducacionais podem estar envolvidos por profissionais de diferentes áreas da saúde, fornecendo um trabalho interdisciplinar o qual fornece ao paciente um atendimento cuja integralidade se faz presente.

\section{Referências}

American Psychiatric Association. (2002). DSM-IV-TR - Manual Diagnóstico e Estatístico de Transtornos Mentais (4. ed.). Porto Alegre, RS: Artmed.

Alcântara, T. V., Shioga, J. E. M., Lima, M. J. V., Lage, A. M. V., \& Maia, A. H. N. (2013). Intervenções psicológicas na sala de espera: Estratégias no contexto da oncologia pediátrica. Sociedade Brasileira de Psicologia Hospitalar, 16(2), 103-119. 
Andretta, I., \& Oliveira, M. S. (2011). A entrevista motivacional em adolescentes usuários de droga que cometeram ato infracional. Psicologia: Reflexão e Crítica, 24(2), 218-226.

Authier, J. (1977). The Psychoeducation Model: Definition, contemporary roots and content. Canadian Journal of Counselling and Psychotherapy, 12(1), 15-22.

Bhattacharjee, D., Rai, A. K., Singh, N. K., Kumar, P., Munda, S. K., \& Das, B. (2011). Psychoeducation: A measure to strengthen psychiatric treatment. Delhi Psychiatric Journal, 14(1), 33-39.

Chien, W. T. (2008). Effectiveness of Psychoeducation and Mutual Support Group Program for Family Caregivers of Chinese People with Schizophrenia. The Open Nursing Journal, 2, 28-39.

Cole, H. P., \& Lacefield, W. E. (1982). Theories of learning, development, and psychoeducational design: Origins and applications in nonschool settings. Viewpoints in Teaching and Learning, $58(3), 6-16$.

Gomes, J. A. L., \& Pergher, G. K. (2010). A TCC no pré e pós operatório de cirurgia cardiovascular. Revista Brasileira de Terapias Cognitivas, 6(1), 173-194.

Gonçalves, R. A., Porto, P. R., Araújo, N. B., Luz, M. P., Figueira, I., \& Ventura, P. (2010). Impacto da co-terapia no tratamento do TEPT com Terapia cognitivo-comportamental. Revista Brasileira de Terapias Cognitivas, 6(1), 32-41.

Gonçalves-Pereira, M., \& Sampaio, D. (2011). Psicoeducação familiar na demência: da clínica à saúde pública. Revista Portuguesa de Saúde Pública, 29(1), 3-10. doi:10.1016/S08709025(11)70002-5

Lau, S., Lu, X., Balsamo, L., Devidas, M., Winick, N., Hunger, S. P., Carroll, W., ...Kadan-Lottick, N. (2015). Family life events in the first year of acute lymphoblastic leukemia therapy: A Children`s Oncology Group Report. Pediatric Blood Cancer, 61(12), 2277-2284. doi:10.1002/ pbc. 25195

Lima-Silva, T. B., \& Yassuda, M. S. (2012). Treino cognitivo e intervenção psicoeducativa para indivíduos hipertensos: Efeitos na cognição. Psicologia: Reflexão e Crítica, 25(1), 30-40.

Lopes, L. O., \& Cachioni, M. (2012). Intervenções psicoeducacionais para cuidadores de idosos com demência: Uma revisão sistemática. Jornal Brasileiro de Psiquiatria, 61(4), 252-261.

Lopes, L. O., \& Cachioni, M. (2013a). Cuidadores familiares de idosos com doença de Alzheimer em uma intervenção psicoeducacional. Revista Brasileira e Geriatria e Gerontologia, 16(3), 443-460.

Lopes, L. O., \& Cachioni, M. (2013b). Impacto de uma intervenção psicoeducacional sobre o bemestar subjetivo de cuidadores de idosos com doença de Alzheimer. Temas em Psicologia, 21(1), 165-181. doi:10.9788/TP2013.1-12

Menezes, S. L., \& Mello e Souza, M. C. B. (2011). Grupo de psicoeducação no transtorno afetivo bipolar: Reflexão sobre o modo asilar e o modo psicossocial. Revista Escola de Enfermagem USP, 45(4), 996-1001. doi:10.1590/S008062342011000400029

Menezes, S. L., \& Mello e Souza, M. C. B. (2012). Implicações de um grupo de Psicoeducação no cotidiano de portadores de Transtorno Afetivo Bipolar. Revista Escola de Enfermagem USP, 46(1), 124-131. doi:10.1590/S008062342012000100017

Mussi, S. V., Soares, M. R. Z., \& Grossi, R. (2013). Transtorno bipolar: Avaliação de um Programa de Psicoeducação sob o enfoque da Análise do Comportamento. Revista Brasileira de Terapia Comportamental e Cognitiva, 15(2), 45-63.

Oliveira, M. I. S. (2011). Intervenção cognitivo-comportamental em transtorno de ansiedade: Relato de Caso. Revista Brasileira de Terapias Cognitivas, 7(1), 30-34.

Petersen, C. S. (2011). Evidências de efetividade e procedimentos básicos para Terapia Cognitivo-Comportamental para crianças com transtorno de ansiedade. Revista Brasileira de Psicoterapia, 13(1), 39-50.

Ponciano, E. L. T., Cavalcanti, M. T., \& Féres-Carneiro, T. (2009). Observando os grupos multifamiliares em uma instituição psiquiátrica. Revista Psiquiatria Clínica, 37(2), 43-47.

Rahmani, F., Ranjbar, F., Ebrahimi, H, \& Hosseinzadeh, M. (2015). The effects of Group Psychoeducational Programme on attitude toward mental illness in families of patients with schizophrenia, 2014. Journal of Caring Sciences, 4(3), 243-251.

Ruzanna, Z., Marhani, M., Parveen, K., \& Cheah, Y. C. (2010). Does psychoeducation improve 
insight of patients with schizophrenia? MJP Online Early.

Sampaio, F. M. C. (2011). A psicoeducação e a sobrecarga dos cuidadores informais do idoso com demência: Análise baseada na evidência. Revista Portuguesa de Enfermagem de Saúde Mental, 5, 30-36.

Scemes, S., Wielenska, R. C., Savoia, M. G., \& Bernik, M. (2011). Fobia de deglutição: Discussão analítico-comportamental de seus determinantes e da amplitude de mudança. Revista Brasileira de Terapia Comportamental e Cognitiva, 13(1), 37-51.

Sousa, S., Marques, A., Curral, R., \& Queirós, C. (2012). Atitudes estigmatizantes em familiares de pessoas com esquizofrenia: Um estudo utilizando o Attribution Questionnaire AQ-27. Trends Psychiatry Psychotherapy, 34(4), 186197.
Tabeleão, V. P., Tomasi, E., \& Quevedo, L. A. (2014). Sobrecarga de familiares de pessoas com transtorno psíquico: Níveis e fatores associados. Revista de Psiquiatria Clínica, 41(3), 63-66.

Wielenska, R. C., \& Banaco, R. A. (2010). Síndrome da fadiga crônica: A perspectiva analítico-comportamental de um caso clínico. Temas em Psicologia, 18(2), 415-424.

Wood, M. M., Brendro, L. K., Fecser, F. A., \& Nichols, P. (1999). Psychoeducation: An Idea Whose Time Has Come. Richmond, VA: The Council for Children with Behavioral Disorders.

Recebido: $27 / 07 / 2015$

$1^{a}$ revisão: 04/01/2016

Aceite final: 14/01/2016 